\title{
A panel discussion
}

\section{Occupational hazards of anaesthesia}

\section{Participants}

W.D.B. Pope MD FRCP(C)

Department of Anesthesia, University of Manitoba (Chairman)

M.J. Halsey D PHIL

Division of Anaesthesia, Clinical Research

Centre, Harrow, England (Environmental Hazards)

G. Hammond MD FRCP(C)

Department of Medical Microbiology, University of Manitoba (Hepatitis, Herpes and AIDS)

C.F. Ward MD

Department of Anesthesia, University of California, San Diego, California (The Impaired Physician)

\section{Introduction}

Over the past 20 years, anaesthetists have begun to realize that their specialty presents a number of important physical and psychological risks to themselves. It is now apparent that daily operating room exposure puts anesthetists at an increased risk in a number of novel and previously unsuspected ways.

Over a decade ago, studies began to appear indicating that women who work in the operating room during pregnancy are more prone to spontaneous abortions and to delivering children with congenital abnormalities than are female health care professionals who do not work in this environment.

Based on presentations given at the Annual Meeting of the Canadian Anaesthetists' Society in Winnipcg,

Manitoba, June 25, 1984.
This culminated in the 1974 report from an Ad Hoc Committee of the American Society of Anesthesiologists which stimulated a great deal of research and eventually resulted in the U.S.A. in the establishment of regulations for maximum allowable levels for inhalation anaesthetics in the operating room air. The corollary to this was the introduction into most North American operating rooms of scavenging devices for these gases.

Dr. Halsey addresses the question of the potential cellular changes resulting from chronic exposure to trace concentrations of anaesthetics and updates our knowledge concerning a possible mechanism for these changes.

Furthermore we tend to have frequent and almost inevitable contact with certain blood products and body secretions from our patients. It is now apparent that this exposure can produce not only morbidity, but also mortality if we deal with these substances without taking careful and in some cases stringent precautions.

Dr. Hammond discusses the present knowledge with regard to diseases of viral aetiology to which our risk may be increased. The prevalence of Hepatitis B in the general population, coupled with changing lifestyles, has made all of us aware of the dangers of exposure to blood products and saliva. This subject and the protective value of immunization procedures will be discussed. As well, information is presented about herpes simplex virus and AIDS, two problems that have become standard material these days for the popular press.

It is now being recognized that anaesthetists undergo psychological stresses and strains which frequently lead to the use of various chemical substances which may seriously impair health and the ability to practice their profession. Dr. Ward discusses our responsibilities towards our 
colleagues in both recognizing and dealing with the impaired physician. More importantly, he discusses therapy for these individuals and indicates the chance of their returning to a functional practice of medicine.

\section{Environmental hazards}

This presentation is intended to convey a personal view of some of the environmental hazards associated with anaesthesia. The hazards include (a) obstetric problems, (b) liver and kidney disease and various cancers and (c) neurological deficits particularly those associated with nitrous oxide.

\section{Obstetric problems}

This is the most widely recognised environmental hazard of anaesthesia but individual opinions, as well as governmental and professional reactions, differ widely. Some feel that not enough is being done about the problem while others maintain either that there is no problem and that much research and finance has been misdirected, or that scavenging devices introduce an unacceptable hazard to the patient. In general many feel that this is a well worn subject about which there is considerable indifference and confusion amongst general operating room staff.

The epidemiological data has been reviewed before (e.g. Vessey and Nunn ${ }^{1}$ ). New information includes the recent Swedish survey (Axelsson and Rylander ${ }^{2}$ ) as well as the Finnish study of obstetrical hazards associated with other aspects of hospital work (Hemminki et al ${ }^{3}$ ).

The animal studies have two contributions to make to the solution of the problem. First, they provide evidence for a cause and effect relationship based on exposure to specific drugs. Second, where there is a satisfactory animal model, it may be possible to determine rational criteria for maximum "safe" levels of contamination. Good examples of the two objectives are provided respectively by the current studies with isofiurane (Mazze et $a l .^{4}$ ) and nitrous oxide (Sharer et al..$^{5}$ ).

\section{Non-obstetric risks}

The information on other health hazards is at the moment less than adequate to allow any firm conclusions. Most British anaesthetists are easily persuaded that disease of the lumbar spine is an occupational hazard associated with transferring patients in the operating room. However, there are serious limitations to the self-reporting of diseases. Various studies have implied that chronic exposure to anaesthetics is associated with a 1.6-1.7-fold increase in liver disease, and a 1.2-1.7-fold increase in kidney disease (Cohen et al. ${ }^{6}$ ) while a morbidity study of British doctors revealed no unusual pattern among anaesthetists compared with other specialists except in increased number of deaths from cancer of the pancreas (Doll and Peto ${ }^{7}$ ). It may be relevant to many occupational groups to note that approximately 50 per cent of pancreatic cancers are thought to be attributable to coffec drinking, a relative risk of 2.7 associated with three or more cups daily (MacMahon et al. ${ }^{8}$ ). A more objective assessment of morbidity would be provided by a study of early retirement from medical practice but it appears that the information required is not easily available.

\section{Neurological deficit associated with nitrous oxide} Nitrous oxide is the only inhalational anaesthetic which undergoes a conventional chemical reaction in vivo. It oxides the cobalt ion of vitamin $\mathrm{B}_{12}$ which results in inactivation of this cofactor in folate related metabolic pathways (Nunn and Chanarin $^{9}$ ). One of the consequences is a condition resembling subacute combined degeneration of the spinal cord, which has been reported in at least 15 people who had abused nitrous oxide (Layser ${ }^{10}$ ). This is probably related to methionine depletion following reduced activity of methionine synthase, the enzyme responsible for recycling methionine and which requires vitamin $B_{12}$ as a bound cofactor.

Methionine plays a crucial role in the synthesis of thymidine, one of the bases of deoxyribonucleic acid (DNA). This is almost certainly the cause of megaloblastic anaemia and leucopenia which follows prolonged administration and could also explain the fetotoxic effects of nitrous oxide associated with chronic exposure to low concentrations. Recent evidence suggests that these potential toxicities have a complex concentration and dose depen- 